\title{
Facile Five-Step Heteroepitaxial Growth of GaAs Nanowires on Si Substrates and the Twin Formation Mechanism
}

Maoqing Yao ${ }^{\dagger, \|}$, Chunyang Sheng ${ }^{\ddagger}, \|$, Mingyuan $\mathrm{Ge}^{\S}$, Chun-Yung $\mathrm{Chi}^{\dagger}, \|$, Sen $\mathrm{Cong}^{\dagger,}$, Aiichiro Nakano ${ }^{\ddagger, \|}$, P. Daniel Dapkus ${ }^{\dagger, \| *}$ and Chongwu Zhou ${ }^{\dagger, \| *}$

${ }^{\dagger}$ Ming Hsieh Department of Electrical Engineering, ${ }^{\ddagger}$ Collaboratory for Advanced Computing and Simulations, Department of Physics and Astronomy, Department of Computer Science, and Department of Chemical Engineering and Materials Science,

${ }^{\S}$ Mork Family Department of Chemical Engineering and Materials Science, ${ }^{\|}$Center for Energy Nanoscience, University of Southern California, Los Angeles, California 90089, United States

\section{Corresponding Author}

*E-mail: (C.Z.) chongwuz@usc.edu

*E-mail: (P.D.D.) dapkus@ usc.edu

\section{Supporting Information}




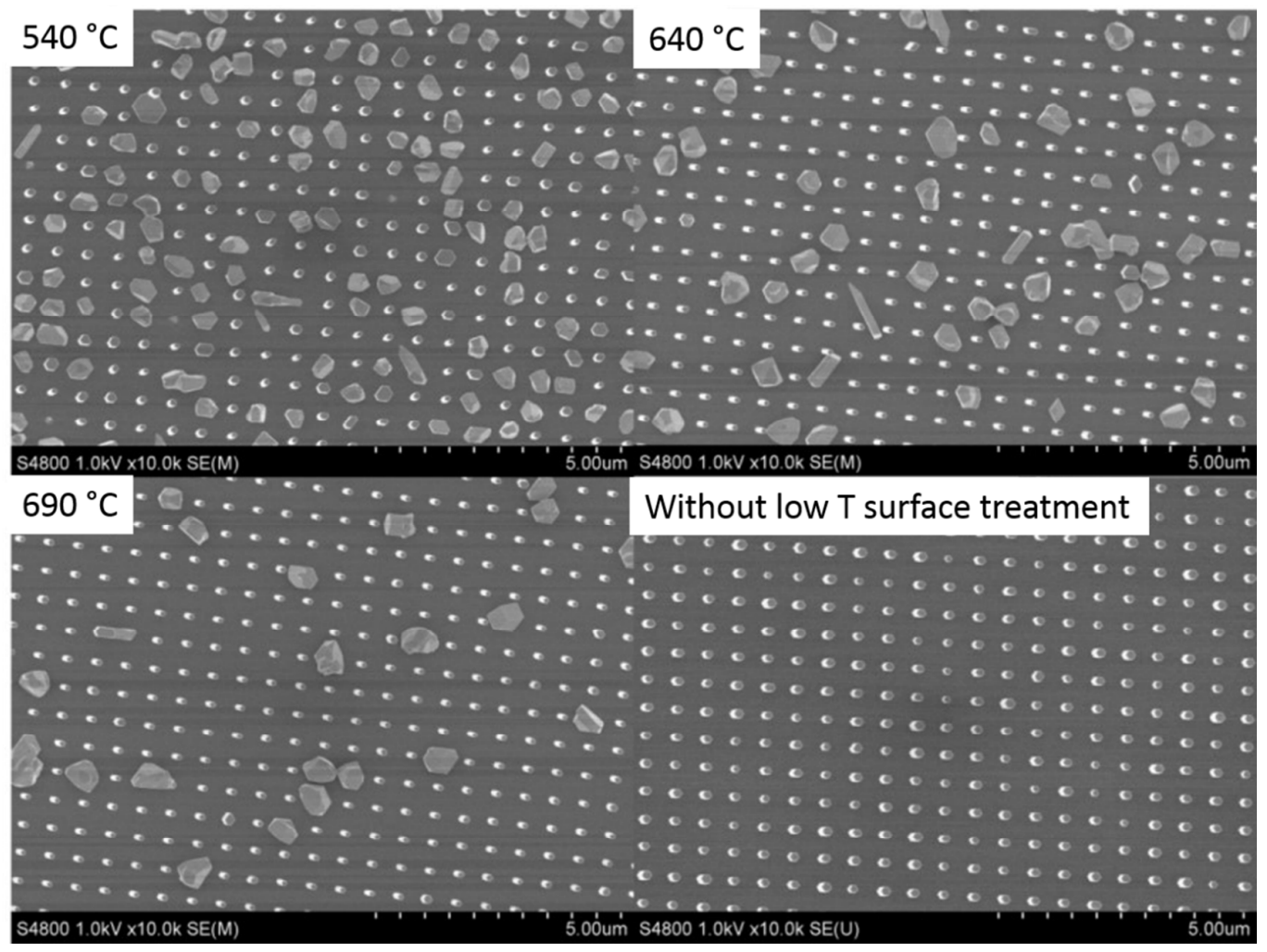

Figure S1. Top view SEM images of nanowire arrays grown with and without low

surface treatment step prior to the nanowire growth. Surface treatment step is 4 minutes if there is. 


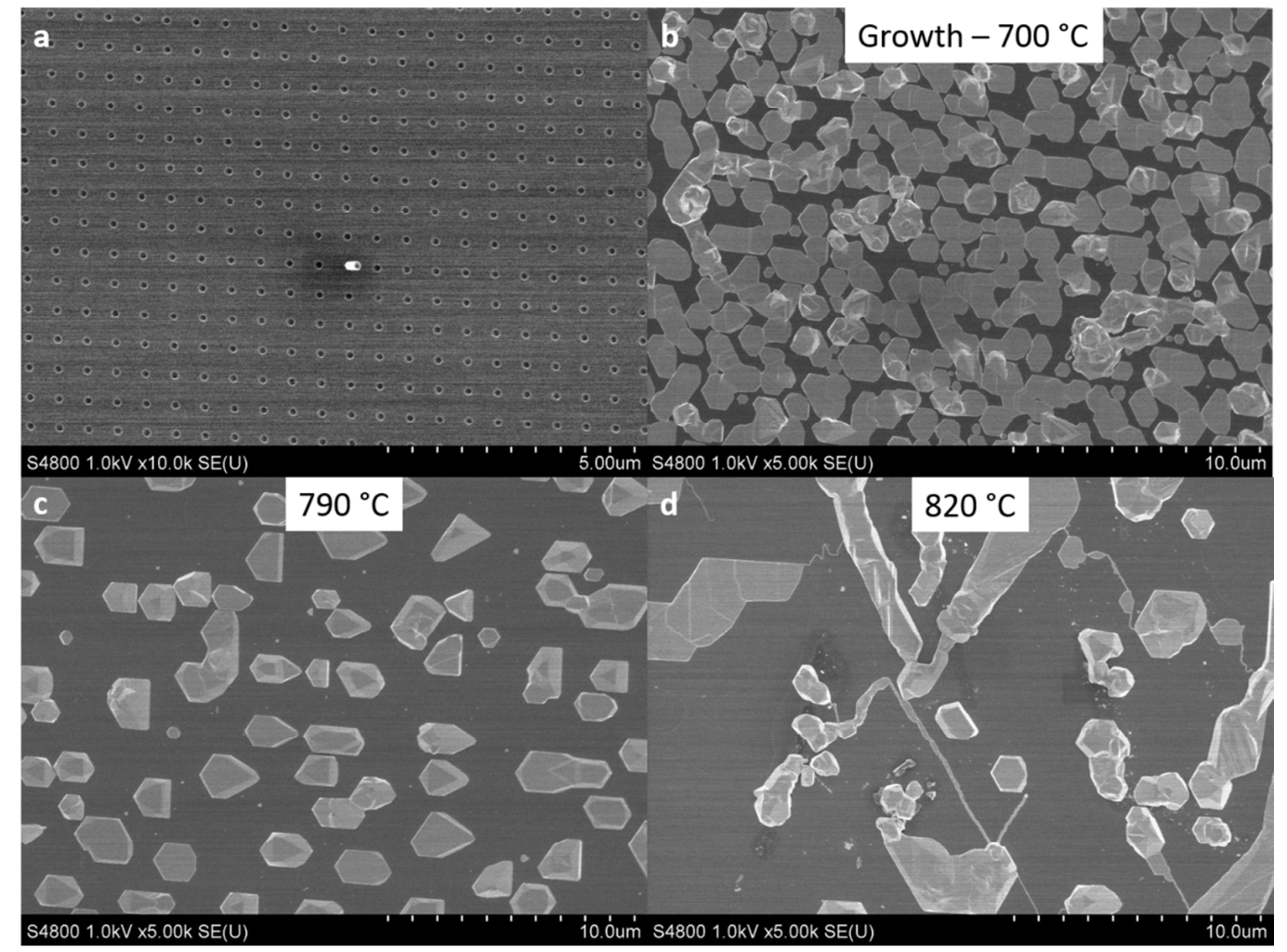

Figure S2. (a) Top view SEM image of sample after growth at $820^{\circ} \mathrm{C}$. Few nanowires can be obtained. (b) - (d) Top view SEM images of bare Si region after nanowire growth at (b) $700{ }^{\circ} \mathrm{C}$, (c) $790{ }^{\circ} \mathrm{C}$ and (d) $820{ }^{\circ} \mathrm{C}$. Island density decreased due to increased Ga diffusion length. In (d) we can see the trace left by large mobile Ga balls. 


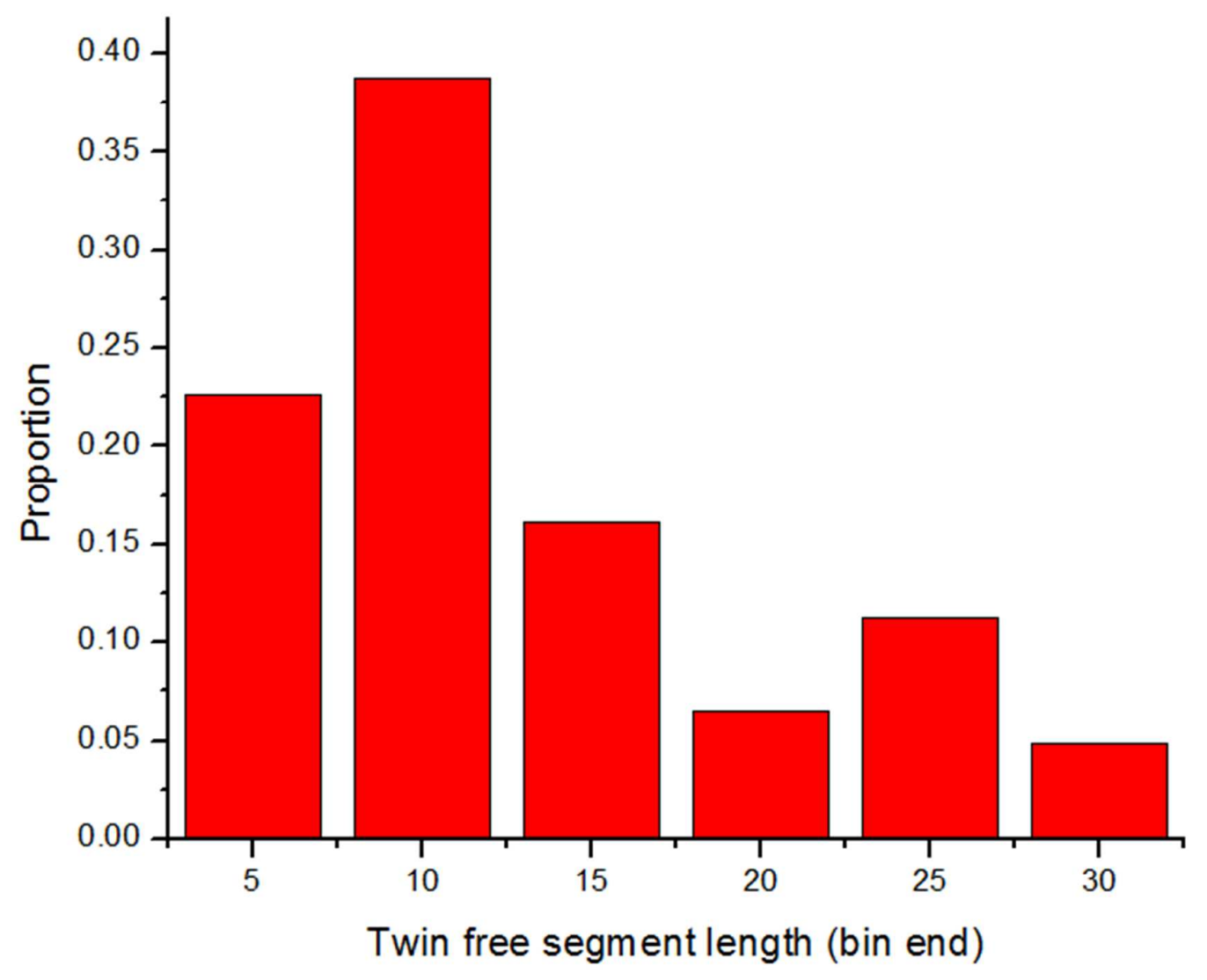

Figure S3. Distribution of twin free segment length in nanowires grown at $790{ }^{\circ} \mathrm{C}$ shown in Figure 4b of main text. 


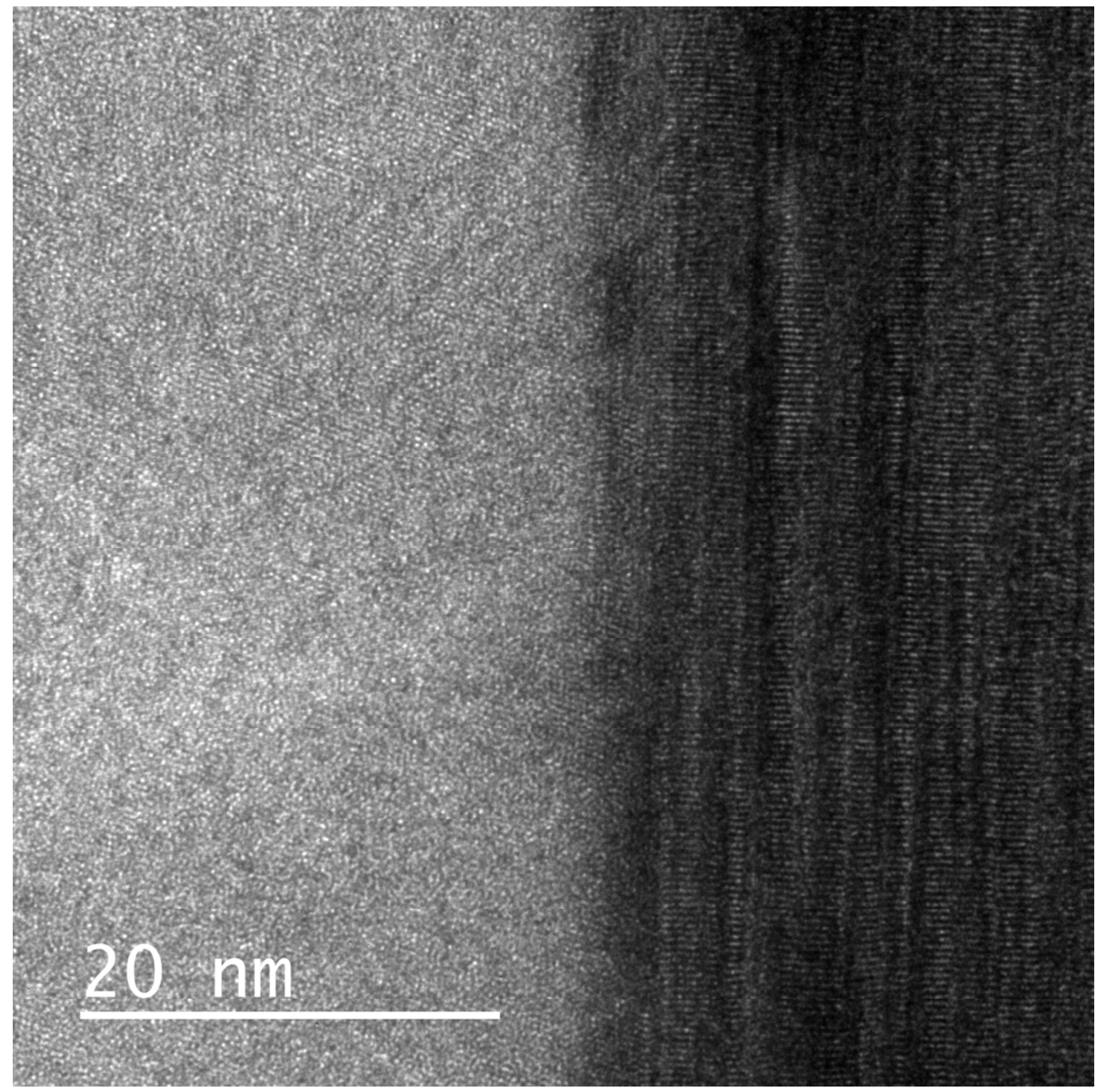

Figure S4. High resolution TEM image at the interface of Si and GaAs of the sample shown in Figure $4 c$ in the main text. The initial part of GaAs nanowire is grown at $760{ }^{\circ} \mathrm{C}$. At some location the twin density is as high as every monolayer, so the crystal structure is effectively Wurtzite. 


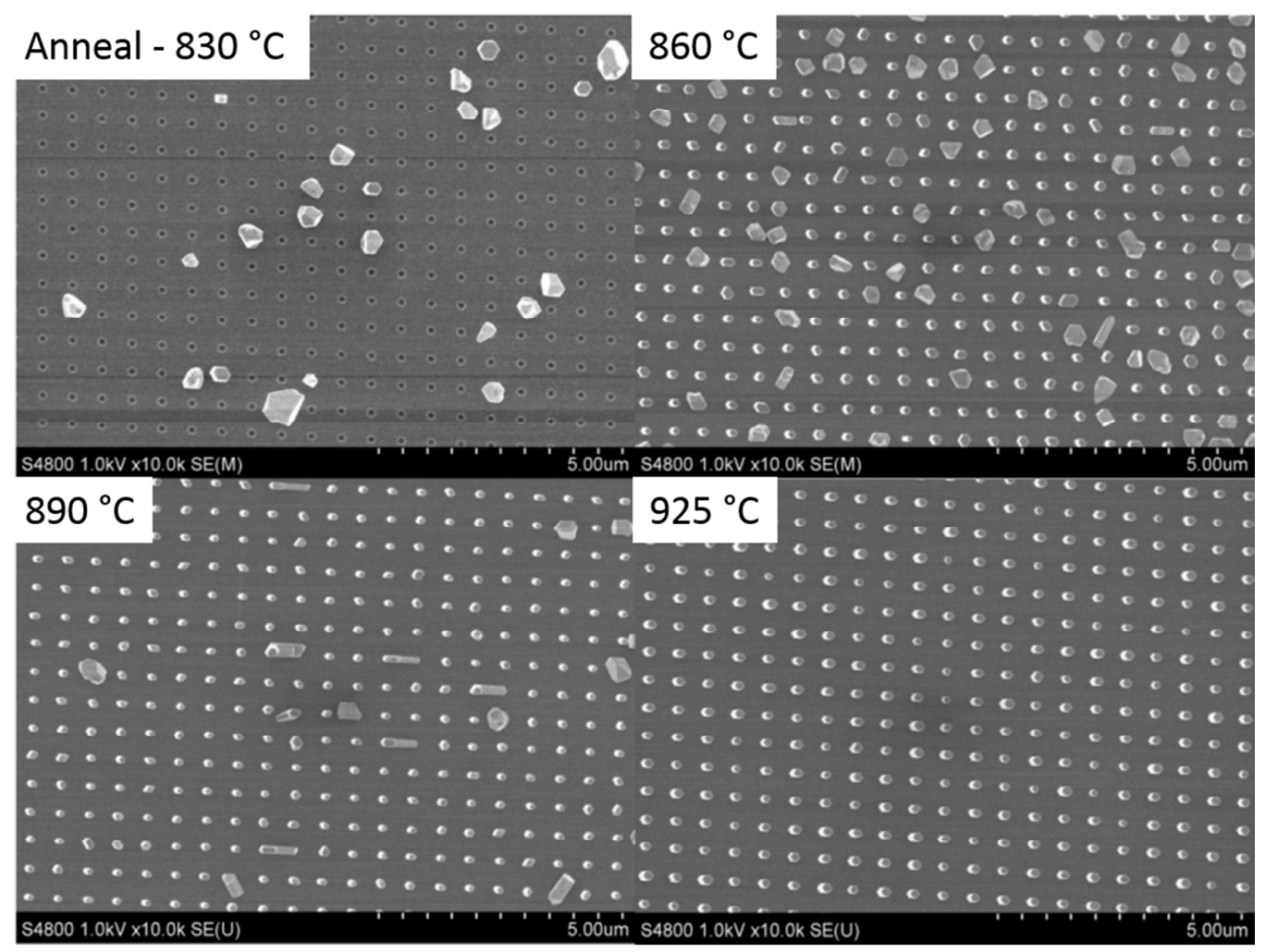

Figure S5. Top view SEM images of GaAs nanowire arrays grown on $\mathrm{Si}$ with

different hydrogen annealing temperatures. Other conditions are all the same as described in text. Lower annealing temperatures reduce vertical nanowire yield 